\title{
Measurement of the radiative branching ratio for the Hoyle state using cas- cade gamma decays
}

\author{
B. Alshahrani ${ }^{1,2, a}$, T. Kibédi ${ }^{1}$, A.E. Stuchbery ${ }^{1}$, E. Williams ${ }^{1}$, and S. Fares ${ }^{3,4}$ \\ ${ }^{1}$ Department of Nuclear Physics, RSPE, The Australian National University, ACT 0200, Australia \\ ${ }^{2}$ Department of Physics, King Khaled University, Abha, Kingdom of Saudi Arabia \\ ${ }^{3}$ Department of Physics, Al-Baha University, Al-Baha, Kingdom of Saudi Arabia \\ ${ }^{4}$ Department of Radiation Physics, National Center for Radiation Research and Technology (NCRRT), Nasr City, Cairo, Egypt
}

\begin{abstract}
A new setup consisting of four 5" by 5" NaI scintillators and an array of particle detectors is being developed. Proton-gamma-gamma coincidences will be measured using the ${ }^{12} C\left(p, p^{\prime}\right)^{12} C$ reaction at $10.5 \mathrm{MeV}$ energy. The new setup will be used for the measurement of the electromagnetic decay rate of the Hoyle state via two E2 transitions. We report the initial experimental results for singles gamma and gamma-proton coincidences through inelastic scattering of protons on a ${ }^{12} \mathrm{C}$ target.
\end{abstract}

\section{Introduction}

It is well known that carbon is produced in the universe by the triple-alpha reaction in helium-burning red giant stars. In 1953, Fred Hoyle realised that the fact that any significant carbon in the Universe requires a resonant state in ${ }^{12} \mathrm{C}$ very near $7.7 \mathrm{MeV}$.

The subsequent observation of this state, known as the Hoyle state, is often cited as the beginning of experimental nuclear astrophysics [1]. The first observation of the Hoyle state was made by Noel Dunbar in 1953 and has been described as the most important experiment performed by an Australian physicist [2].

The structure of the Hoyle state is difficult to explain. It is generally believed to be based on $\alpha$-clusters in a linear chain structure [3], however an alternative explanation of the $\alpha$ clustering in analogy to ultra-cold gases has been proposed [4]. Recent studies show that, based on ab initio lattice calculations, the Hoyle state has a structure like a compact triangular configuration of alpha clusters [5]. A somewhat different picture emerged from the analysis of the proton and $\alpha$ scattering data [6] suggesting that the $\alpha$ particles form an open triangle shape or can be considered as a loose assembly. However all recent studies exclude the possibility of a linear chain structure.

Our project aims to improve the knowledge of the radiative width of the Hoyle state through the observation of the cascading gamma-rays, the $3.21 \mathrm{MeV}$ and $4.44 \mathrm{MeV}$ transitions. One should note that there is only one experimental spectrum [7] ever published showing the electromagnetic decay branch from the Hoyle state. We report the initial experimental results from the detections of singles gamma and gamma-proton coincidences through the inelastic scattering of protons on a ${ }^{12} \mathrm{C}$ target.

\footnotetext{
ae-mail: badriah.alshahrani@anu.edu.au
}

\section{$23 \alpha$ reaction}

The triple-alpha reaction has a significant role in the production of carbon in the universe. The $3 \alpha$ process and the formation of ${ }^{12} \mathrm{C}$ are illustrated in Fig. 1. It is one of the most important reactions in the field of nuclear astrophysics.

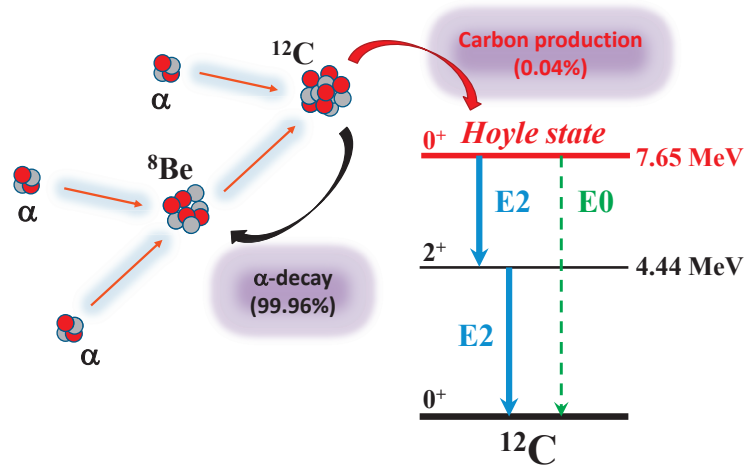

Figure 1. $3 \alpha$ process and the formation of ${ }^{12} \mathrm{C}$.

We can describe the $3 \alpha$ reaction as follows: helium nuclei fuse into heavier elements when the temperature is high enough, typically around $10^{8} \mathrm{~K}$, and when the helium density is in the order of $10^{5} \mathrm{~g} / \mathrm{cm}^{3}$ [8]. Two helium nuclei fuse to form ${ }^{8} \mathrm{Be}$ (an unstable isotope with short half-life of $\left.5 \times 10^{-17} \mathrm{sec}\right) .{ }^{12} \mathrm{C}$ is created when a third alpha particle fuses with the ${ }^{8} \mathrm{Be}$ nucleus, and the unstable Hoyle state is populated, which has a short half-life around $2.4 \times 10^{-16} \mathrm{~s}$. The chain of this process is as follows:

$$
\begin{array}{cc}
{ }^{4} \mathrm{He}+{ }_{\text {Step I }}^{4} \mathrm{He} \leftrightarrow{ }^{8} \mathrm{Be} & { }^{4} \mathrm{He}+{ }^{8} \mathrm{Be} \leftrightarrow \\
{ }^{12} \mathrm{C}^{*}
\end{array}
$$


The Hoyle state decays via internal transitions $0.04 \%$ of the time and decays via $\alpha$ emission to ${ }^{8} \mathrm{Be} 99.96 \%$ of the time.

The rate of the triple-alpha reaction can be written as $r_{3 \alpha} \propto \Gamma_{\text {rad }} \exp \left(-Q_{3 \alpha} k T\right)$, where $T$ is the temperature, $Q_{3 \alpha}$ is the energy released in the ${ }^{12} \mathrm{C}(7.65 \mathrm{MeV}) \rightarrow 3 \alpha$ decay and $\Gamma_{\text {rad }}$ is the radiative width. The uncertainty of the triple-alpha reaction rate comes mainly from the uncertainty in $\Gamma_{\text {rad }}$.

\section{The decay of the Hoyle state}

The detection and the isolation of the relevant $\gamma$-rays with high efficiency is a key to studying the decay of the Hoyle state (Fig. 1). Two paths of decay to the ground state are possible: through the 3.21 and $4.44 \mathrm{MeV}$ cascade $\gamma$-rays, and through the direct 7.65 MeV E0 transition. The 3.21 $\mathrm{MeV}$ transition proceeds mainly $(99.9 \%)$ via $\gamma$-photon emission. The remaining rate occurs by pair conversion.

The total rate of ${ }^{12} \mathrm{C}$ producing decays from the Hoyle state depends directly on the radiative width, $\Gamma_{\text {rad }}$, which includes the width for $\gamma$ emission $\left(\Gamma_{\gamma}\right)$, internal conversion $\left(\Gamma_{\mathrm{CE}}\right)$, and pair production $\left(\Gamma_{\pi}\right)$; i.e.

$$
\Gamma_{\mathrm{rad}}=\Gamma_{\gamma}^{\mathrm{E} 2}+\Gamma_{\pi}^{\mathrm{E} 0}+\Gamma_{\pi}^{\mathrm{E} 2}+\Gamma_{\mathrm{CE}}^{\mathrm{E} 0}+\Gamma_{\mathrm{CE}}^{\mathrm{E} 2} .
$$

Thus, in summary, the largest contribution to the uncertainty of $r_{3 \alpha}$ comes from $\Gamma_{r a d}$ which is the sum of the partial decay widths for the photon $(98.5 \%)$, pair conversion $(1.5 \%)$ and electron conversion $(<0.01 \%)$.

\section{New setup to improve the radiative branching ratio}

The radiative width $\Gamma_{\text {rad }}$ is usually determined from three experimental quantities (shown in square brackets) [9]:

$$
\Gamma_{\mathrm{rad}}=\left[\frac{\Gamma_{\mathrm{rad}}}{\Gamma}\right] \times\left[\frac{\Gamma}{\Gamma_{\pi}^{\mathrm{E} 0}}\right] \times\left[\Gamma_{\pi}^{\mathrm{E} 0}\right],
$$

where $\Gamma$ is the total width of the Hoyle state and $\Gamma_{\pi}^{\mathrm{E} 0}$ is the absolute E0 transition width. $\Gamma_{\mathrm{rad}} / \Gamma$ and $\Gamma_{\pi}^{\mathrm{E} 0} / \Gamma$ are the ratio of the radiative and E0 pair conversion width to the total width. The main aim of our study is to improve the accuracy of the first term, $\Gamma_{\mathrm{rad}} / \Gamma$.

A new setup has been developed to improve the accuracy of $\Gamma_{\text {rad }} / \Gamma$, by a factor of 2 . This study complements our project to determine the radiative width from the pair conversion of the E0 and E2 transitions from the Hoyle state [10]. The Hoyle state will be populated in the laboratory using the ${ }^{12} C\left(p, p^{\prime}\right)^{12} C(7.654 \mathrm{MeV})$ reaction with 10.5 MeV protons from 14UD Heavy Ion Accelerator Facility of the Australian National University.

The set up consists of four $5^{\prime \prime}$ by $5^{\prime \prime}$ sodium iodide (NaI) scintillator detectors mounted at $\pm 45^{\circ}$ and $\pm 135^{\circ}$ angles to the beam direction and about $11 \mathrm{~cm}$ from the target. The new setup is shown in Fig. 2.

The $\gamma$-ray detectors are combined with eight surface barrier detectors (SB). Each particle detector has an active area of $10 \mathrm{~mm}$ by $10 \mathrm{~mm}$ with an effective thickness of about $100 \mu \mathrm{m}$. The mean proton detection angle is about $150^{\circ}$. The beam collimator is made from Tantalum located $80 \mathrm{~cm}$ upstream and shielded by $2.5 \mathrm{~cm}$ lead.

The data were collected event by event and written onto disk for offline analysis. Each event consists of a proton and two $\gamma$-ray energies in coincidence, as well as their associated times, which allows for the subtraction of random coincidences. An event is defined as follows: inelastic scattering of the incoming proton excites the Hoyle state, which then decays via two photons of E2 multipolarity to the ground state. The ratio of such events compared to the total excitation of the Hoyle state determines the $\Gamma_{\text {rad }} / \Gamma$ branching ratio.

\begin{tabular}{|c|c|}
\hline Reference, Reaction and Method & $\Gamma_{\mathrm{rad}} / \Gamma \times \mathbf{1 0}^{-4}$ \\
\hline $\begin{array}{l}\text { Alburger }(1961)[11] \\
{\left[{ }^{10} B\left({ }^{3} \mathrm{He}, p\right){ }^{12} \mathrm{C}\right]} \\
\text { p } \gamma \gamma \text { coinc }\end{array}$ & $3.3(9)$ \\
\hline $\begin{array}{l}\text { Seeger \& Kavanagh (1963) } \\
{\left[{ }^{14} N(d, \alpha){ }^{12} C\right]} \\
\quad \text { Recoiling }{ }^{12} C \text { and } \alpha \text { coinc }\end{array}$ & $2.82(29)^{(1)}$ \\
\hline $\begin{array}{l}\text { Hall \& Tanner (1964) } \\
{\left[{ }^{10} B\left({ }^{3} \mathrm{He}, p\right){ }^{12} \mathrm{C}\right]} \\
\quad \text { Recoiling }{ }^{12} \mathrm{C} \text { and } \mathrm{p} \text { coinc }\end{array}$ & $3.5(12)$ \\
\hline $\begin{array}{l}\text { Chamberlin et al. (1974) } \\
{\left[{ }^{12} C\left(\alpha, \alpha^{\prime}\right)^{12} C\right]} \\
\text { Inelastic } \alpha \text { and } \\
\text { associated }{ }^{12} C \text { coinc }\end{array}$ & $4.2(2)$ \\
\hline $\begin{array}{l}\text { Davids et al. (1975) } \\
{\left[{ }^{12} C\left(p, p^{\prime}\right)^{12} C\right]} \\
\quad \text { Recoiling }{ }^{12} C \text { and p coinc }\end{array}$ & $4.30(20)$ \\
\hline $\begin{array}{l}\text { Mak et al. }(1975)[16] \\
{\left[{ }^{13} C\left({ }^{3} \mathrm{He}, \alpha\right){ }^{12} \mathrm{C}\right]} \\
\quad \text { Recoiling }{ }^{12} \mathrm{C} \text { and } \alpha \text { coinc }\end{array}$ & $4.15(34)$ \\
\hline $\begin{array}{l}\text { Markham et al. (1976) [17] } \\
{\left[{ }^{12} C\left(\alpha, \alpha^{\prime}\right)^{12} C\right]} \\
\text { Recoiling }{ }^{12} C \text { and } \alpha \text { coinc }\end{array}$ & $3.87(25)$ \\
\hline $\begin{array}{l}\text { Obst et al. }(1976)[7] \\
{\left[{ }^{12} C\left(p, p^{\prime}\right){ }^{12} C\right]} \\
\text { p } \gamma \gamma \text { coinc }\end{array}$ & $4.09(29)$ \\
\hline Adopted & 4.13(11) \\
\hline
\end{tabular}

Table 1. Experimental values of $\Gamma_{\text {rad }} / \Gamma$.

Many attempts have been made to determine $\Gamma_{\mathrm{rad}} / \Gamma$ from measurements. Two of the previous experimental groups $[7,11]$ have been used p $\gamma \gamma$ coincidences, while the rest have used the associated-particle technique with different reactions. These are listed in Table. 1. The adopted

${ }^{1}$ Datum was excluded from the analysis. 
value of $\Gamma_{\mathrm{rad}} / \Gamma$ has been obtained from the experimental values included in Table. 1, excluding the second value which is considred to be an outlier. Credible results were obtained in the study of Obst and Braithwaite [7] more than 35 years ago by using proton- $\gamma-\gamma(\mathrm{p} \gamma \gamma)$ coincidence. An essential element of this $\mathrm{p} \gamma \gamma$ coincidence measurement was the subtraction of random coincidence events under various timing conditions.

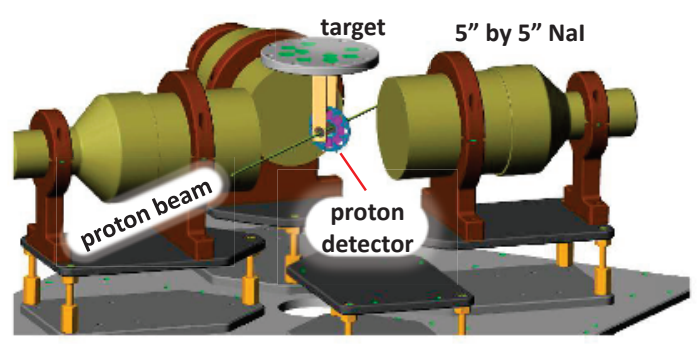

Figure 2. The proton $-\gamma-\gamma$ coincidence setup based on four $5 "$ by $5 " \mathrm{NaI}$ detectors and an array of proton detectors. One of the $\mathrm{NaI}$ detectors and the vacuum chamber are not shown (Courtesy of Alan Harding, ANU).

\section{Initial gamma-proton measurements}

Initial test experiments have been carried out with 10.5 $\mathrm{MeV}$ protons incident on a $200 \mu \mathrm{g} / \mathrm{cm}^{2}$ thick ${ }^{12} \mathrm{C}$ target, using the ${ }^{12} C\left(p, p^{\prime}\right)^{12} C$ reaction. For the energy calibration a ${ }^{56} \mathrm{Co}$ source was used for the $\gamma$ detectors and $\alpha$ particles from a ${ }^{241} \mathrm{Am}$ source were used for the particle detectors.

Figure 3 shows the singles gamma-ray spectrum recorded in the present experiment using one of the $\mathrm{NaI}$ scintillator detectors. This spectrum is compared with a spectrum recorded with a high resolution, Compton suppressed $\mathrm{HpGe}$ spectrometer using the same reaction. The spectra are dominated by the $4.44 \mathrm{MeV}$ E2 transition from ${ }^{12} \mathrm{C}$. Additional high energy transitions are also observed from the ${ }^{16} \mathrm{O}$ contamination in the target. Distinct features of the high-energy lines include the single- and doubleescape peaks.

In the first experiment, proton-gamma coincidence events were collected and representative spectra are shown in Figure 4. The total projected particle spectrum is show in the insert. This spectrum has three proton groups. According to reaction kinematics, the peak at around 4.3 $\mathrm{MeV}$ and $1.2 \mathrm{MeV}$ can be attributed to the excitations of the $4.44 \mathrm{MeV}$ and the $7.765 \mathrm{MeV}$ states in ${ }^{12} \mathrm{C}$. The third proton group is expected at $7.8 \mathrm{MeV}$ from the elastic scattering of the $10.5 \mathrm{MeV}$ bombarding particles on the target. The silicon detectors used in this experiment are not expected to stop such high energy protons, and it is expected that they will be observed as an energy-loss peak.

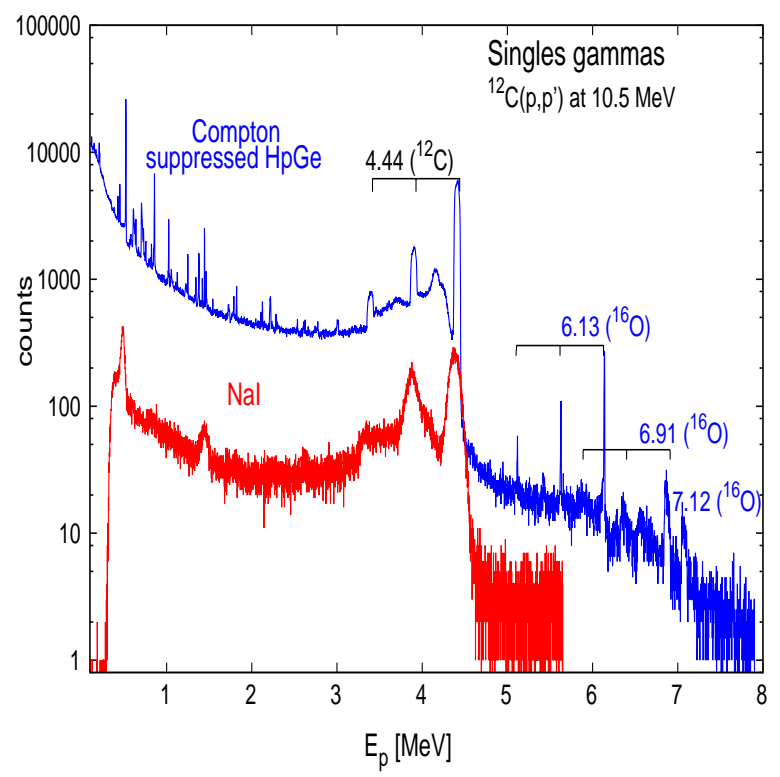

Figure 3. Singles $\gamma$-ray spectra recorded by Compton suppressed HpGe detectors (blue) and by NaI scintillator detectors (red).

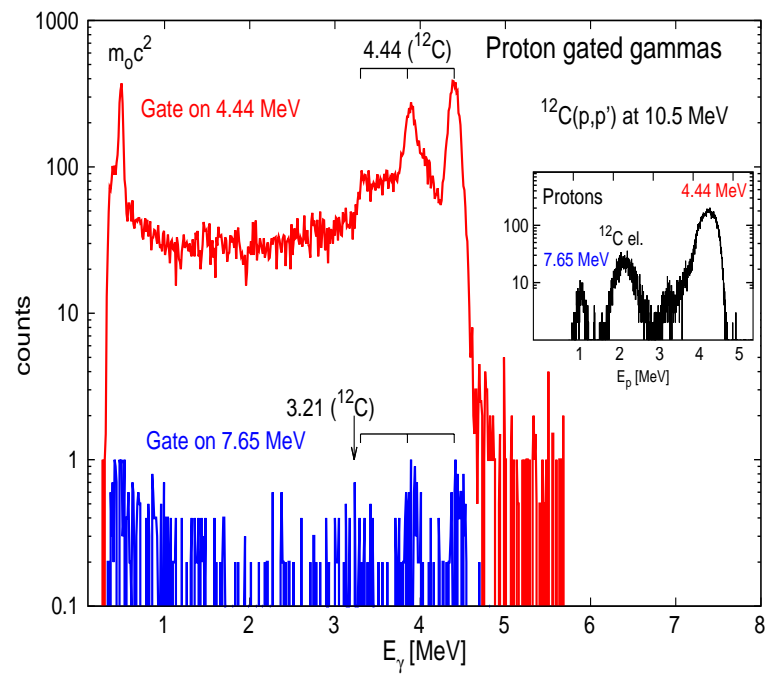

Figure 4. Proton gated gamma spectra recorded with the NaI scintillator detectors. Gate on $4.44 \mathrm{MeV}$ (red) and gate on 7.65 $\mathrm{MeV}$ (blue) proton groups.

This assumption was further explored with Monte Carlo simulations using the SRIM code [18]. In these calculations the energy deposit of the $1.2,4.3$ and $7.8 \mathrm{MeV}$ energy-protons was evaluated for different detector thicknesses, ranging from $50 \mu \mathrm{m}$ to $200 \mu \mathrm{m}$. While protons are fully stopped up to an energy of $4.3 \mathrm{MeV}$, the mean energy of the $7.8 \mathrm{MeV}$ protons depends on the detector thickness. Satisfactory agreement with the experimental data was achieved for a detector thickness of about $100 \mu \mathrm{m}$. 
Representative gamma-ray spectra, gated by the 4.3 $\mathrm{MeV}$ (labelled as $4.44 \mathrm{MeV}$ ) and $1.2 \mathrm{MeV}$ (labelled as $7.65 \mathrm{MeV}$ ) protons, respectively, are shown in Figure 4. While the first spectrum is expected to be dominated by the $4.44 \mathrm{MeV}$ E2 transition, the second spectrum should contain the two cascading E2 transitions at $3.21 \mathrm{MeV}$ and 4.44 MeV energy. This spectrum only contains a few events, possibly from chance proton-gamma coincidences. An arrow indicates, where the $3.21 \mathrm{MeV}$ E2 transition deexciting the Hoyle state is expected.

\section{Conclusion}

We described the methods and the apparatus required to measure the $\Gamma_{\mathrm{rad}} / \Gamma$ branching ratio with an uncertainty better than the current adopted value of $4.13(11) \times 10^{-4}$, which is an average over eight measurements [7].

Four large volume sodium iodide $(\mathrm{NaI})$ scintillator detectors were used in combination with particle detectors and event-by-event data collection.

Further measurements with improvements are required for the suppression of a low energy $\leq 1 \mathrm{MeV}$ photon background, to reduce the Compton scattering of photons from one detector to the other by using shielding between them, to evaluate proton spectra recorded with the silicon photodiodes, and to calibrate for singles protons and $p \gamma \gamma$ coincidences.

\section{References}

[1] D.N.F. Dunbar, R.E. Pixley, W.A. Wenzel, and W. Whaling, Phys. Rev. 92 (1953) 3.

[2] R. Spear, The Physicist 39 (2002) 35.

[3] H. Morinaga et al., Phys. Rev. 101, (1956) 254; Phys. Lett. 21 (1966) 78.

[4] M. Kamimura et al., Nucl. Phys. A 351 (1981) 456.

[5] E. Epelbaum et al., Phys. Rev. Lett. 109 (2012) 252501.

[6] M. Freer et al., Phys. Rev. C86 (2012) 034320.

[7] A.W. Obst, W.J. Braithwaite, Phys. Rev. C13 (1976) 203.

[8] E.E. Salpeter, Phys. Rev. 107 (1957) 16.

[9] S.M. Austin, Nucl. Phys. A758 (2005) 375c.

[10] T. Kibédi et al., Proc. of the 14th Int. Symp. on Capture Gamma-Ray Spectroscopy and Related Topics, Guelph, Canada, World Scintific, (2013) p.328.

[11] D.E. Alburger, Phys. Rev. 124 (1961) 193.

[12] P.A. Seeger, R.W. Kawanash, Astrophys. J. 137 (1963) 704.

[13] I. Hall and N.W. Tanner, Nucl. Phys. 53 (1964) 673.

[14] D. Chamberlin et al., Phys. Rev. C9 (1974) 69.

[15] C.N. Davids et al., Phys. Rev. C11 (1975) 2063.

[16] H.B. Mak et al., Phys. Rev. C12 (1975) 1158.

[17] R.G. Markham et al., Nucl. Phys. A270 (1976) 489.

[18] J.F. Ziegler, M.D. Ziegler, J.P. Biersack, (SRIM.com) (2008). 